\title{
Тепловые эффекты при кристаллизации \\ и плавлении сплавов с неограниченной растворимостью компонентов
}

\author{
Л. В. Спивак ${ }^{a}$, Е. А. Сюткина \\ Пермский государственный национальный исследовательский университет \\ 614990, Пермь, ул. Букирева, 15 \\ a email: lspivak2@mail.ru \\ b email: enya1995@yandex.ru
}

\begin{abstract}
Методом дифференциальной сканирующей калориметрии высокого разрешения (DSC) исследованы процессы кристаллизации двухкомпонентных сплавов с неограниченной растворимостью образующих их компонентов. Показано, что во всех случаях непосредственно ниже линии ликвидус фиксируется заметный по величине и скорости скачок выделения тепла кристаллизации. Предполагается, что обнаруженные эффекты связаны с образованием в жидкости при приближении к линии ликвидус значительного количества локальных микрообъемов (концентрационных флуктуаций), обогащенных ведущим кристаллизацию компонентом. Их появление предшествует началу процессов спонтанного образования большого числа кристаллов в заметном объеме жидкой фазы.
\end{abstract}

Ключевые слова: кристаллизация; дифференциальная сканирующая калориметрия; двухкомпонентные сплавы

Поступила в редакциию 26.11.2018; принята к опубликованию 19.03.2019

\section{Thermal effects in the crystallization and melting of alloys with unlimited solubility of components}

\author{
L.V. Spivak ${ }^{a}$, E.A. Syutkina ${ }^{b}$ \\ Perm State University, Bukireva 15, 614990, Perm, Russia \\ ${ }^{\text {a }}$ E-mail: lspivak2@mail.ru \\ b E-mail: enya1995@yandex.ru
}

High-resolution differential scanning calorimetry (DSC) has been used to study the crystallization processes of two-component alloys with unlimited solubility of the components that form them. It is shown that in all cases, immediately below the liquidus line, a jump in the release of crystallization heat that is noticeable in magnitude and speed is recorded. It is assumed that the observed effects are associated with the formation of a significant amount of local microvolumes (concentration fluctuations) in the liquid when the liquidus line is approaching the liquidus line, enriched with the component leading to crystallization. Their appearance precedes the onset of the processes of spontaneous formation of a large number of crystals in a noticeable volume of the liquid phase.

Keywords: crystallization; differential scanning calorimetry; two-component alloys

Received 26.11.2018; accepted 19.03.2019

doi: 10.17072/1994-3598-2019-1-47-51

(C) Спивак Л. В., Сюткина Е. А., 2019 


\section{1. Введение}

Процессы кристаллизации металлов и присущие им закономерности достаточно давно стали предметом экспериментальных и теоретических исследований. Однако анализ литературных данных, представленных в широко известных обзорах [1-3], показывает, что описанию процессов кристаллизации двухкомпонентных сплавов уделяется по сравнению с кристаллизацией однокомпонентных жидкостей очень скромное внимание. Некоторым исключением следует считать рассмотрение в этих работах эвтектического превращения.

С появлением в последнее время аппаратуры DSC появилось возможность анализа явлений, протекающих при кристаллизации двухкомпонентных расплавов в интервале температур между линиями ликвидус и солидус. Однако известные нам исследования с использованием DSC-метода при кристаллизации сплавов относятся к многокомпонентным сплавам, при изучении которых основное внимание уделялось технологическим аспектам их применения [4-6]. Некоторым исключением из этого является исследование, проведенное на сплавах системы Al-Si [6]. Но и в этом случае акцент был сделан на выяснение влияния модификаторов на структуру таких двухкомпонентных сплавов.

Поэтому важно выяснить физику процессов, происходящих в межкритическом интервале температур, для чего возможно проведение исследования кристаллизации и плавления двухкомпонентных сплавов систем $\mathrm{Ag}-\mathrm{Au}$ и $\mathrm{Cu}-\mathrm{Au}$ методом DSC. Они могут рассматриваться как модельные для сплавов с неограниченной растворимостью образующих их компонентов.

\section{2. Методика исследования}

Объектом исследования служили сплавы систем $\mathrm{Ag}-\mathrm{Au}, \mathrm{Cu}-\mathrm{Au}$. Данные по дифференциальной сканирующей калориметрии были получены с помощью прибора STA «Jupiter» 449 фирмы Netzsch. Нагрев и охлаждение производили со скоростью 5, 10, 20 и 40 К/мин в атмосфере аргона (99.999\% Ar). Скорость потока газа 25-30 мл/мин. Обработка экспериментальных данных по DSC peализована с использованием программного обеспечения «Proteus Analyses» и пакета «Fityk».

Для исключения возможности появления при исследовании сплавов артефактов, обусловленных особенностями эксперимента, проводили сглаживание экспериментальных данных полиномом пятой степени с точностью аппроксимации не менее 95\%. Этот же прием использовался и при анализе данных по первой производной (dDSC) сигнала DSC по температуре.

\section{3. Экспериментальные результаты и их обсуждение}

На рис. 1 показаны типичные DSC зависимости при охлаждении расплавов компонентов с неограниченной растворимостью друг в друге и последующем нагреве сплава при плавлении. Качественно аналогичные зависимости установлены и для сплавов системы $\mathrm{Cu}-\mathrm{Au}$. Судя по диаграммам состояния сплавов $\mathrm{Cu}-\mathrm{Au}$ (рис. 1) и $\mathrm{Ag}-\mathrm{Au}$ (рис. 2), в них наблюдается весьма малое расстояние между линиями ликвидус и солидус, что и определяет, как известно, их хорошие литейные свойства. Данные DSC также подтверждают эту особенность. Межкритический интервал для сплава $\mathrm{Ag}-\mathrm{Au}$ составил около $10{ }^{\circ} \mathrm{C}$, для сплава $\mathrm{Cu}-\mathrm{Au}$ $-15^{\circ} \mathrm{C}$. Для обоих сплавов на зависимости DSC от температуры между линиями ликвидус и солидус наблюдается асимметричный экзотермический пик. Асимметричность пиков связана с тем, что для начала процесса кристаллизации нужна более низкая температура (переохлаждение), чем для процесса затвердевания. Вид первой производной dDSC свидетельствует (см. рис. 3) о скачке скорости выделения тепла по достижению или чуть ниже линии ликвидус.

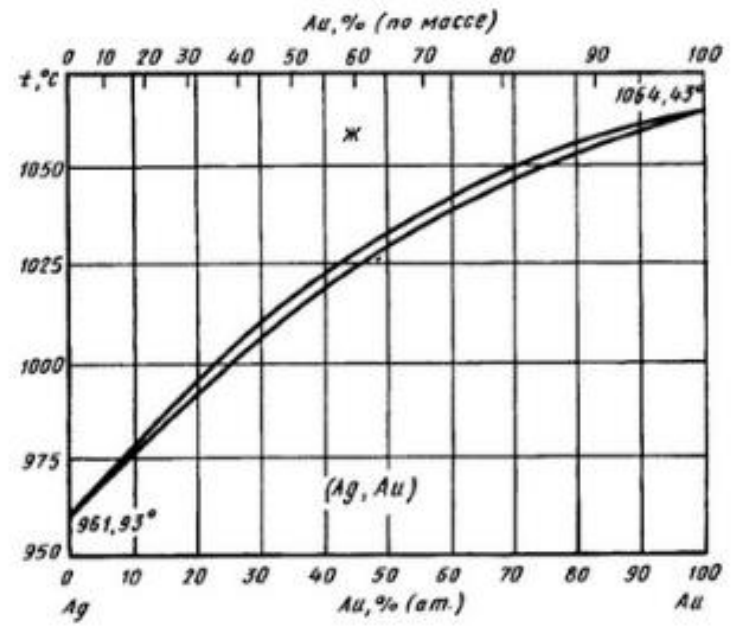

Рис. 1. Диаграмма состояния двухкомпонентного сплава системы $A g-A u$

Величина теплового эффекта в этом случае должна определяться тепловым эффектом превращения и объемной долей жидкой фазы, которая участвует в превращении (объемная скорость превращения). Как видно из представленных данных, сразу же после перехода линии ликвидус наблюдается значительный тепловой поток.

Согласно правилу отрезков, при переходе линии ликвидус не должно выделяться заметное количество кристаллической фазы. Следовательно, такой скачок теплового потока не должен был бы наблюдаться. По-видимому, традиционные представления о развитии кристаллизационных про- 
цессов в межкритическом интервале температур нуждаются в определенной корректировке.

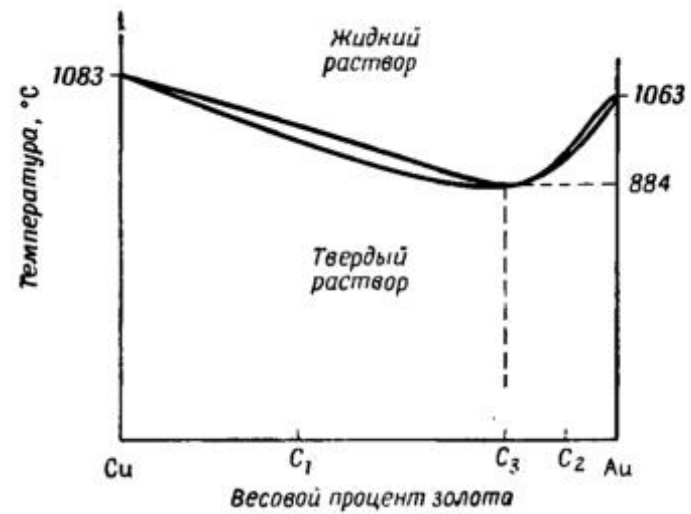

Рис. 2. Диаграмма состояния двухкомпонентного сплава системы $\mathrm{Cu}-\mathrm{Au}$

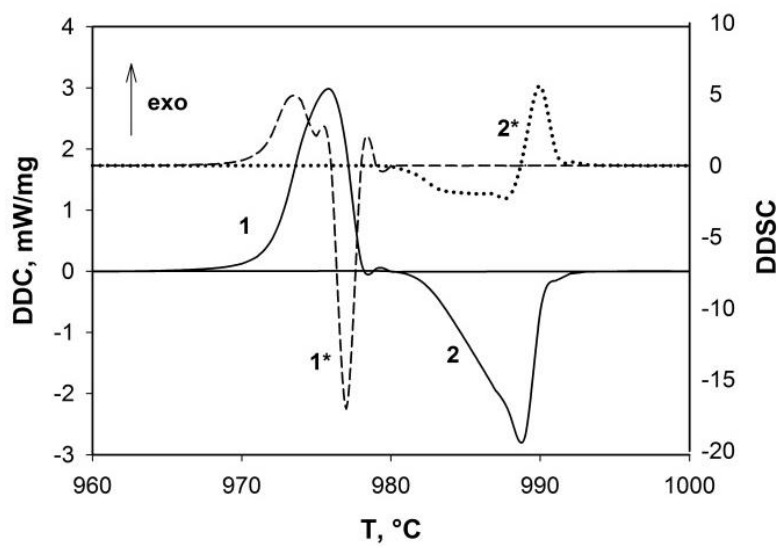

Рис. 3. Зависимость сигнала DSC (1) $u$ dDSC (2) при кристаллизачии и плавлении сплава Au-Ag (22.7\% Au). Скорость нагрева $5 \mathrm{~K} /$ мин

Показано (см., например, рис. 4), что экзотермические пики на DSC зависимостях при охлаждении двухкомпонентных расплавов с неограниченной растворимостью компонентов можно представить как суперпозицию двух подпиков. Последнее указывает на реализацию двух механизмов кристаллизации расплава при его охлаждении между линиями ликвидус и солидус. Как видно из представленных данных, один из таких подпиков (Р1) расположен в верхнем интервале температур между линиями ликвидус и солидус. Он в большинстве случаев описывается функцией с резким правым фронтом (SplitPearson7). Другой подпик (P2), более симметричный, характеризует процессы, протекающие во всем температурном интервале кристаллизации.

По-видимому, высокотемпературный подпик в структуре экзотермического пика отражает процессы зарождения кристаллической фазы, тогда как второй подпик - рост ранее возникших кристаллов.
Можно предложить следующее объяснение установленным закономерностям. Для того чтобы из жидкости началось выделение кристаллов, состав которых заметно отличается от среднего состава компонентов в жидком состоянии, в этой жидкости при приближении к линии ликвидус должно произойти расслоение на области с относительно низкой и относительно высокой, близкой к составу возникающих кристаллов, концентрацией одного из компонентов. Происходит нечто близкое к спинодальному распаду пересыщенных твердых растворов [7].

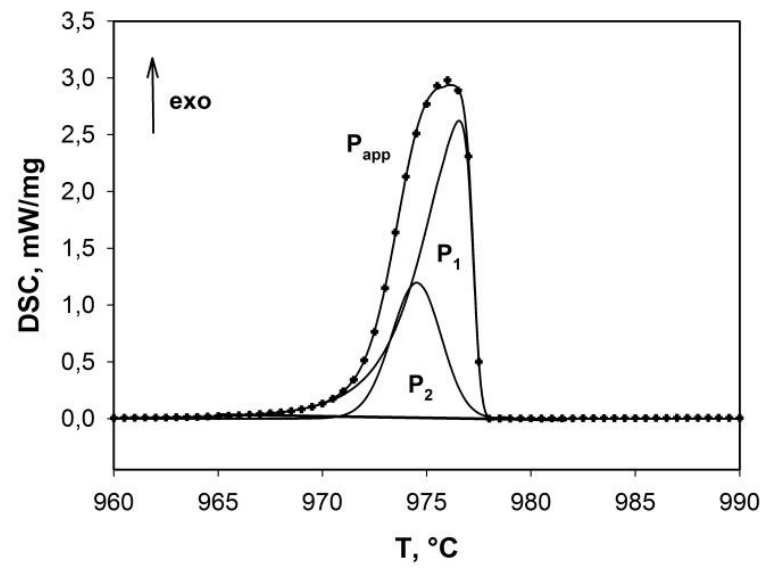

Рис. 4. Строение экзотермического пика на рис. 1: точки - экспериментальные данные; $P_{\text {арр }}$ - результат аппроксимаџии; $P_{1} u P_{2}-$ подпики. Скорость нагрева $5 \mathrm{~K} /$ мин

При переходе линии ликвидус в таких уже «подготовленных» к возникновению новой фазы объемах спонтанно происходит образование большого числа центров кристаллизации. В этот процесс вовлекается заметная часть кристаллизующейся жидкости. В совокупности, как предполагается, это и приводит к интенсивному тепловыделению при переходе линии ликвидус.

В контексте сказанного выше целесообразно остановиться на одной из последних попыток теоретического определения скорости зарождения центров кристаллизации, которая осуществлена Чалмерсом $[1,2]$. Предполагается, что при переохлаждении $\sim 0.2 \mathrm{~T}_{\mathrm{s}}$ наблюдается быстрое возрастание скорости образования зародышей. Результаты применения метода диспергирования образцов кристаллизующейся жидкости на капли микроскопического размера были обобщены Торнбаллом [1] и для большинства жидкостей $\Delta \mathrm{T}_{\max }>0.15$ Ts. Для ГЦК и ОЦК металлов $\Delta \mathrm{T}_{\max } \sim 0.18 \mathrm{Ts}$. Это достаточно близко к теоретическому анализу ситуации.

Обзоры по затвердеванию жидкостей, опубликованные в последнее время (см. [2, 3]), незначительно изменили представления о скорости зарождения центров кристаллизации.

Принципиальной особенностью таких исследований по определению скорости образования цен- 
тров кристаллизации и скорости их последующего роста является то, что они предполагают проведение изотермических экспериментов при заданной величине переохлаждения $\Delta T$. Но и в этих монографиях практически не рассмотрены процессы при кристаллизации двухкомпонентных сплавов. Приведенные же данные по кристаллизации эвтектических расплавов касаются не столько скорости зарождения новых кристаллов, а сколько скорости роста ячеек с чередующимися выделениями двух одновременно кристаллизующихся фаз (см., например, [2, 3]).

На затруднение процесса кристаллизации в тех случаях, когда состав кристаллов отличается от состава жидкой фазы, в свое время обратил внимание Пуассон [8], который ввел представления о существовании концентрационных флуктуаций.

Однако существующие теории кристаллизации достаточно полно охватывают лишь те процессы, в результате которых состав твердой фазы совпадает с составом затвердевающей жидкости: кристаллизацию расплавленных чистых металлов и гомогенных химических соединений. Только в этих случаях вероятность образования равновесного кристаллического зародыша определяется одной лишь необходимостью создания поверхности раздела между твердой и жидкой фазами и равна вероятности энергетической флюктуации.

Таким образом, не только при кристаллизации однокомпонентных жидкостей, но и, в первую очередь, при кристаллизации двухкомпонентных расплавов вблизи температуры ликвидус должно происходить расслоение жидкой фазы на огромное число кластеров, структура которых и соотношение компонентов в которых близки к таковым для возникающей кристаллической фазы. Как следствие, при переходе линии ликвидус в значительном объеме жидкой фазы возникают многочисленные кристаллы. Спонтанное появление большого числа таких кристаллов приводит к наблюдаемым калориметрическим эффектам.

Необходимо обратить внимание на еще одну обнаруженную в ходе исследования закономерность. При нагреве двухкомпонентного сплава (см. рис. 3 и 5) наблюдаются сложное строение эндотермического пика, как это было установлено при кристаллизации, и скачок скорости поглощения тепла.

Сложилось впечатление, что кристаллы, возникшие при начале кристаллизации и с появлением которых связан скачок выделения тепла, при нагреве также исчезают спонтанно в том же интервале температур, в котором они образовались при охлаждении.

Увеличение скорости нагрева или охлаждения не приводит к отклонению от рассмотренных закономерностей. Поскольку температурный интервал между линиями ликвидус и солидус менее $20^{\circ}$, то при скорости его прохождения 40 К/мин процесс кристаллизации или плавления занимает ме- нее 3 сек. Это означает, что двухстадийный механизм кристаллизации или плавления имманентно присущ сплавам с неограниченной растворимостью компонентов и не зависит от скорости прохождения межкритического интервала температур.

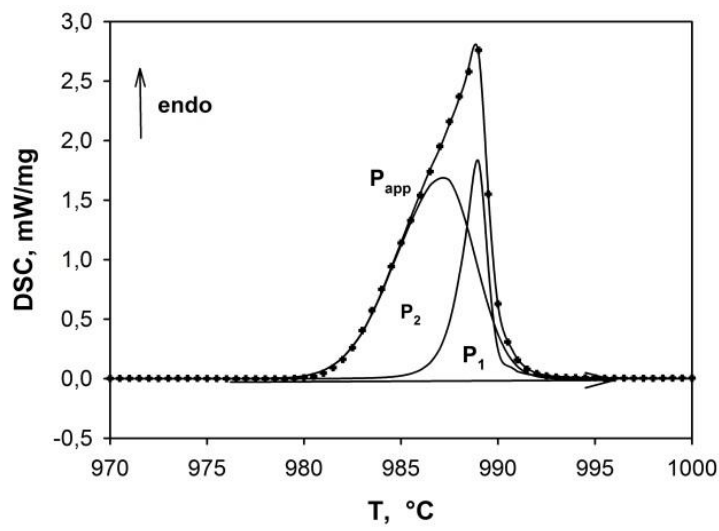

Рис. 5. Строение эндотермического пика на рис. 1: точки - экспериментальные данные; $P_{\text {арр }}$ - результат аппроксимации; $P_{1} и P_{2}$ подпики. Скорость нагрева $5 \mathrm{~K} /$ мин

\section{4. Заключение}

Показано, что при кристаллизации сплавов с неограниченной растворимостью образующих их компонентов вблизи линии ликвидус резко возрастают скорость выделения и величина тепла кристаллизации, которые затухают по мере исчерпания жидкой фазы.

Высказано предположение, что при приближении к линии ликвидус двухкомпонентных расплавов в жидкости происходит образование обогащенных ведущим кристаллизацию компонентом локальных кластеров, появление которых предшествует началу процессов образования и роста кристаллов новой фазы. Скачкообразный характер развития кристаллизационного процесса в значимом объеме жидкости сопровождается активным выделением тепла сразу же после перехода линии ликвидус. При нагреве закристаллизовавшегося сплава процесс перехода из твердой фазы в жидкую также состоит из двух этапов и характеризуется скачком поглощения тепла в близком к линии ликвидус интервале температур.

\section{Список литературы}

1. Biloni H., Boetinger W. J. In physical metallurgy. / Cahn R. W., Haasen P. (Eds.). Amsterdam: NorthHolland, 1996. P. 669-779.

2. Glicksman M. E. Principles of solidification: An introduction to modern casting and crystal growth concepts. New-York: Springer, 2011. 520 p.

3. Kurz W., Fisher D. J. Fundamentals of solidification. Switzerland: Trans. Tech. Publication, 1998. $305 \mathrm{p}$. 
4. Heusler L., Schneider W. Influence of alloying elements on the thermal analysis results of $\mathrm{Al}-\mathrm{Si}$ cast alloys // Journal of Light Metals. 2002. Vol. 2. N. 1. P. 17-26.

5. Piatkowski J., Przeliorz V., Szymszal V. The application of ATD and DSC methods to study of the EN AC-48000 alloy phase transformations // Archives of Foundry Engineering. 2017. Vol. 17 (2). P. 207-211.

6. Piątkowski J., Gajdzik B Testing phase changes in Al-Si alloys with application of thermal analysis and differential calorimetric analysis // MetaBK. 2013. Vol. 52 (4). P. 469-472.

7. Скрипов А. В., Скрипов В. П. Спинодальный распад (фазовый переход с участием неустойчивых состояний) // Успехи физических наук. 1979. Т. 128. С. 193-281.

8. Вентцель Е. С., Овчаров Л. А. Теория вероятностей и её инженерные приложения. М.: Высшая школа, 2000. 480 с.

\section{References}

1. Biloni H., Boetinger W. J. In physical metallurgy. Ed. by Cahn R. W., Haasen P. Amsterdam: NorthHolland, 1996, pp. 669-779.
2. Glicksman M. E. Principles of solidification: An introduction to modern casting and crystal growth concepts. New-York: Springer, 2011520 p.

3. Kurz W., Fisher D. J. Fundamentals of solidification. Switzerland: Trans. Tech. Publication. 1998. $305 \mathrm{p}$.

4. Heusler L., Schneider W. Influence of alloying elements on the thermal analysis results of $\mathrm{Al}-\mathrm{Si}$ cast alloys. Journal of Light Metals, 2002, vol. 2, no. 1, pp. 17-26.

5. Piątkowski J., Przeliorz V., Szymszal V. The application of ATD and DSC methods to study of the EN AC-48000 alloy phase transformations. Archives of Foundry Engineering, 2017, vol. 17 (2), pp. 207-211.

6. Piątkowski J., Gajdzik B Testing phase changes in Al-Si alloys with application of thermal analysis and differential calorimetric analysis. MetaBK, 2013, vol. 52 (4), pp. 469-472.

7. Skripov A.V., Skripov V. P. Spinodal decomposition (phase transitions via unstable states). Soviet Physics Uspekhi, 1979, vol. 22, pp. 389-410.

8. Ventsel E. S., Ovcharov L. A. Probability theory and its engineering applications. Moscow: High School, 2000, 480 p. (In Russian).

\section{Просьба ссылаться на эту статью в русскоязычных источниках следующим образом:}

Спивак Л. В., Сюткина Е. А. Тепловые эффекты при кристаллизации и плавлении сплавов с неограниченной растворимостью компонентов // Вестник Пермского университета. Физика. 2019. № 1. С. 47-51. doi: 10.17072/1994-3598-2019-1-47-51

\section{Please cite this article in English as:}

Spivak L. V., Syutkina E. A. Thermal effects during crystallization and melting of alloys with unlimited solubility of components. Bulletin of Perm University. Physics, 2019, no. 1, pp. 47-51. doi: 10.17072/1994-3598-2019$1-47-51$ 